\title{
How Routinely Assessed Biomarkers Can Be Utilized To Identify Individuals With A High Disease Burden: A Bioinformatics Approach Towards Predictive, Preventive And Personalised (3P) Medicine
}

\section{Nina Bertele}

Stanford University https://orcid.org/0000-0002-5754-2172

Alexander Karabatsiakis ( $\nabla$ alexander.karabatsiakis@uibk.ac.at)

University of Innsbruck

\section{Claudia Buss}

Charité - Universitätsmedizin Berlin, Berlin

\section{Anat Talmon}

Stanford University

\section{Research Article}

Keywords: Biomarkers, biochemical clusters, disease burden, childhood maltreatment, child abuse, personalized medicine, targeted prevention

Posted Date: September 7th, 2021

DOl: https://doi.org/10.21203/rs.3.rs-813085/v1

License: (c) (1) This work is licensed under a Creative Commons Attribution 4.0 International License. Read Full License 


\section{Abstract}

Prevalences of non-communicable diseases such as depression and a range of somatic diseases are continuously increasing requiring simple and inexpensive ways to identify high-risk individuals to target with predictive and preventive approaches. Using k-mean cluster analytics, in study 1 , we identified biochemical clusters (based on C-reactive protein, interleukin-6, fibrinogen, cortisol, and creatinine) and examined their link to diseases. Analyses were conducted in a U.S. American sample (from Midlife in the United States study, $N=1,234$ ) and validated in a Japanese sample (from Midlife in Japan study, $N=$ 378). In study 2 , we investigated the link of clusters to childhood maltreatment (CM). The three identified biochemical clusters included one cluster (with high inflammatory signaling and low cortisol and creatinine concentrations) indicating the highest disease burden. This high-risk cluster also reported the highest CM exposure. The current study demonstrates how biomarkers can be utilized to identify individuals with a high disease burden and thus, may help to target these high-risk individuals with tailored prevention/intervention, towards personalized medicine. Furthermore, our findings raise the question whether the found biochemical clusters have predictive character; as a tool to identify high-risk individuals enabling targeted prevention. The finding that $\mathrm{CM}$ was mostly prevalent in the high-risk cluster provides first hints that the clusters could indeed have predictive character and highlight $\mathrm{CM}$ as a central disease susceptibility factor and possibly as a leverage point for disease prevention/intervention.

\section{Introduction}

Prevalence and incidence of non-communicable diseases (NCD) are continuously increasing in numbers, causing a strong socio-economic as well as a medical burden to the healthcare systems. Economically speaking, the U.S. health care costs have steadily increased for four consecutive years, to reach 3.8 trillion U.S. dollars in 2019 [1,2]. NCD caused $90 \%$ of these costs as they result in massive long-term treatment costs and often present with comorbidities [1,2]. Thus, the prevention of NCD, and in this context the identification of at-risk individuals and sensitive biomarkers of disease risk, is more important than ever as it represents a leverage point to reduce the economic as well as the individual burden of diseases.

The two-consecutive study presented here demonstrates how routinely assessed biomarkers can be bioinformatically clustered and utilized to identify individuals with a high disease burden. Specifically, in study 1 , we employed a clustering approach based on the levels of C-reactive protein (CRP), interleukin-6 (IL-6), fibrinogen, cortisol, and creatinine in a U.S. cohort and validate the identified clusters in a Japanese cohort (for a study overview see Figure 1). We then linked these biochemical clusters to documented diseases including depression, heart disease, hypertension, stroke, peptic ulcer disease (PUD), and cancer. In study 2, we tested the association of childhood maltreatment (CM), a well-established early-life risk factor for developing mental and somatic disorders, with diseases as well as with the identified biochemical clusters from study 1 .

\section{General Methods}




\section{Description of the study populations}

\section{U.S. American Sample}

Data were drawn from the biomarker subsample of the Midlife in the United States (MIDUS) study between 1995 and 1996 [3]. For more information about the project please see [http://www.midus.wisc.edu/data/index.php]. A total of 1,255 individuals participated in the biomarker study, and of those complete biomarker data was available from 1,234 individuals.

\section{Japanese Sample}

Data were drawn from the Midlife in Japan (MIDJA) study ( $N=1,027)$. In 2009-2010 biomarker data were generated for a subset of these participants $(N=378)$. Data were obtained analogically to MIDUS.

\section{Study 1}

\section{Introduction}

According to the Global Burden of Disease study (2017), between 1990 and 2017, disability-adjusted life years (DALYs) due to NCD increased from 1.2 to 1.6 billion. With that, NCD caused more than $60 \%$ of DALYs worldwide [4]. But NCD not only cause individual suffering but also burden society as a whole, due to massive monetary and non-monetary costs $[4,5]$. Relying on interventions -- no matter how effective they are -- after individuals are already ill, is therefore a pivotal fallacy. Instead, current developments require simple and inexpensive ways to identify high-risk individuals to target with both preventive and interventive approaches. Furthermore, it is increasingly becoming clear, that many well-established risk factors (such as Body Mass Index (BMI) outside the normal range [6], genetic risk factors [7,8], etc.) supposedly helping to identify individuals at high risk for certain diseases are not independently from the individual environment and do not behave the same way across different individuals; highlighting the importance of personalized, tailored approaches in the context of preventive medicine. The presence of one particular risk factor might not have much predictive character for negative outcomes without being considered systemically/holistically, that is, in the context of other physiological, environmental, psychological, and biochemical parameters and processes [e.g., 6-8]. Despite these intricacies, at the same time, disease-predictive measures should be cost-efficient making it possible to implement them in the health care system.

One particular concept that has become well-established in the literature is the concept of allostatic load (referring to the cumulative burden of chronic stress and adverse life events) with its suggested allostatic load index (ALI) [9]. ALI is a cumulative multi-system risk score based on physiological and biochemical measures [10]. For each system, risk indices are calculated as the proportion of biomarkers for which an individual falls into predefined high-risk quartiles. 
As a systemic risk score, ALI is predictive for various outcomes, including all-cause mortality $[11,12]$, while there are some critical limitations concerning its conceptualization. First, calculating a risk score as the sum of different system risk scores does not allow to account for intersystemic interactions and the possible predictive effect of these interactions. This gap is unfortunate as ALI includes parameters that indeed are not independent of each other, such as BMI and blood pressure [13]. Another concern refers to practicability and implementation of ALI into the health care system. While ALI considers parameters that can be assessed relatively simple, it is still likely that, for most individuals, parameters are only partially available, limiting the predictive power of ALI. Together, ALI is a profound concept but artificially splits physiological processes that are woven into a holistic allostatic reaction, as acknowledged by the developers of ALI [14]. Furthermore, ALI lacks practicability, which is underlined by the fact that, to date, ALI has not been implemented in routine diagnostics.

Given the rising number of NCD, there is an urgent necessity to develop an approach that is practicable, cost-efficient, and at best, based on biomarkers that are assessed in clinical routine allowing to identify high-risk individuals to target with specific preventive steps. The current study aimed to develop and validate an easily accessible measure that can realistically be implemented in routine diagnostics. Towards this aim and building on ALI, five biomarkers were chosen as they cover broad physiological functionality; CRP, fibrinogen, and IL-6 are pro-inflammatory markers (i.e., positive association with inflammation), cortisol as the end product of the hypothalamus-pituitary-adrenal axis is an immunemodulatory mediator playing a crucial role in stress response, and creatinine is important for cellular energy metabolism [15-19]. Contrary to ALI, employing a clustering approach based on these biomarkers allows to account for linear and non-linear interactions among them and to link resulting clusters to depression and a range of somatic diseases. To examine the association between biochemical clusters and diseases, we focused on depression, heart disease, hypertension, stroke, PUD, and cancer as these represent globally highest prevalence, the fastest increase in numbers, and utmost comorbidities [4]. We first clustered biochemical markers and related them to odds ratios (ORs) for diseases in a U.S. population sample and then repeated this process in a Japanese cohort. To ensure representativity, both samples were recruited via random-digit-dialing qualifying them for studies with results generalizable to the population. Towards our aim to ensure that the selected biomarkers and their clustering demonstrates robust applicability across different cultures and ethnicities [20], we chose one U.S. American and one Japanese sample to generate and validate the biochemical clusters.

\section{Methods}

\section{Collection of Biosamples and the Assessment of Biochemical Markers}

MIDUS. Blood samples were collected after overnight fasting for the assessment of CRP, IL-6, and fibrinogen, according to the manufacturer guidelines (Dade Behring Inc., Deerfield, IL for CRP and fibrinogen; R\&D Systems, Minneapolis, Minnesota for IL-6) [20]. Plasma levels of CRP and fibrinogen were assayed using immunonephelometric assay; IL- 6 was quantitatively assessed using Enzyme-Linked 
Immunosorbent Assay (ELISA). The laboratory inter-assay coefficient of variance was $5.7 \%$ for CRP, $13 \%$ for IL-6, $2.6 \%$ for fibrinogen, all below the $20 \%$ acceptable range [21].

To obtain a cumulative cortisol and creatinine measure 12-hour overnight urine samples were also collected between 7 PM and 7 AM. Enzymatic Colorimetric Assays and Liquid Chromatography-Tandem Mass Spectrometry were performed at the Mayo Medical Laboratory in Rochester, Minnesota. Data were excluded if participants had a renal failure or severe renal decline according to glomerular filtration rate [21].

MIDJA. CRP, IL-6, and fibrinogen were assessed analogically to MIDUS, while cortisol was assessed in saliva (three subsequent days, three times each day) and creatinine was assessed in blood. The 9 saliva measurements were averaged and used as a representative marker for cortisol concentrations [22]. We used blood levels of creatinine.

\section{Diseases}

Depression, heart disease, hypertension, stroke/Transient Ischemic Attack (TIA), PUD, and cancer were assessed via self-report. Participants were asked if they were diagnosed with any of these diseases at timepoint of study participation.

\section{Statistical Analyses}

First, the potential collinearity of the biomarker levels was assessed by calculating Pearson correlations among CRP, fibrinogen, IL-6, creatinine, and cortisol. After randomizing the order of participants [23], we performed a k-mean cluster analysis with these markers in the MIDUS sample using IBM SPSS Statistics 27. To ensure the stability of clusters, we repeated the clustering process in subsamples [23]: Specifically, we conducted a median-split based on age and performed the clustering for each group separately to assess whether the clusters are age-dependent. For the same purpose, we repeated the clustering procedure after excluding participants with a BMI outside the health range (below 18 or above 35 ). The next step was to repeat biochemical clustering, that was performed for the whole MIDUS sample, in the MIDJA cohort. Finally, z-tests were used to compare ORs for diseases among clusters.

\section{Results}

\section{Preliminary Analyses}

In both MIDUS and MIDJA samples, biomarkers were positively correlated (see SI Tables 4 and 5).

In MIDUS, $24.1 \%$ of the participants (currently or previously) had depression, $11.5 \%$ heart disease, $37.1 \%$ hypertension, $4.3 \%$ stroke/ TIA, 5.3\% PUD, and $13.6 \%$ cancer. In MIDJA, $4.5 \%$ of the participants had depression, $5.6 \%$ heart disease, $19.3 \%$ hypertension, $1.1 \%$ stroke/TIA, 8.3\% PUD, and $5.1 \%$ cancer.

\section{K-Mean Clustering}


We used $z$-standardized biomarkers for k-mean clustering and evaluated the clustering results from $\mathrm{k}=2$ to 6 clusters for MIDUS. When $\mathrm{k}=2$, the patterns of clusters were not distinct enough; when $\mathrm{k}=$ 4 or above, some clusters were very small in size (i.e., smallest cluster portion: $8 \%$ ). Through a combination of the parsimonious principle and engineering meaningful difference among clusters, $\mathrm{k}=3$ were selected for the subsequent analyses. Figure 2 illustrates the distributions of the three identified clusters with respect to the biochemical markers. We replicated all three clusters in the younger MIDUS cohort as well as clusters 1 and 2 in the older MIDUS cohort (SI Figures 7 and8). We further replicated all three clusters in the BMI-restricted MIDUS cohort (SI Figure 9).

Then, the 3-cluster solution from MIDUS was validated in the MIDJA sample; the results are shown in Figure 3.

As depicted in Figures 2 and 3, cluster 1 is characterized by average levels in all biochemical measures. Cluster 2 is characterized by high and above-average levels oforCRP, IL- 6 and fibrinogen. Cluster 3 is characterized by high and above-average levels for cortisol and creatinine but average levels for CRP, fibrinogen, and IL-6.

\section{Associations between biochemical clusters and disease states}

MIDUS. Cluster 2 had the highest ORs for all considered diseases compared to the clusters 1 and 3 (Figure 4, SI 10).

MIDJA. Cluster 3 had the highest ORs for heart disease, hypertension, and PUD, cluster 2 had the highest ORs for stroke and cancer, and cluster 1 had the highest ORs for depression (Figure 5).

To compare this cluster-based approach to a well-established clinical biomarker that is associated with a broad range of NCD, the number of diagnoses among individuals in cluster 2 was compared to the number of diagnoses among individuals with CRP concentrations above the clinical cut-off $(>3 \mathrm{mg} / \mathrm{L})$ [24]. The disease burden in cluster 2 was higher with 1.6 diagnoses $(S D=1.16 ; 0.9$ diagnoses for individuals not assigned to cluster 2) compared to individuals above the CRP-cutoff with 1.2 diagnoses $(S D=1.07 ; 0.9$ diagnoses for individuals below the cutoff).

\section{Discussion}

Findings reveal three distinct and interculturally stable biochemical clusters observable in the general population. Cluster 1 is characterized by average levels of all biomarkers, cluster 2 by high inflammationrelated mediators coupled with low cortisol and creatinine, and cluster 3 by high levels of cortisol and creatinine. The stability of clusters is supported by their replication in the MIDJA sample as well as in the BMI-restricted, in the younger (below age median) and in the older MIDUS cohort (above age median; here only clusters 1 and 2 were replicated). However, we did not replicate cluster 3 in the older MIDUS cohort. One explanation could be that, due to an age-related increase in systemic inflammation [25], older individuals were not assigned to cluster 3 , which is characterized by low inflammation. 
Relating clusters to diseases, in MIDUS, cluster 2 showed the highest ORs for depression, heart disease, hypertension, stroke, and cancer (Figure 4). These findings are supported by previous evidence suggesting that CRP, IL-6, and fibrinogen are associated with depression [26,27], coronary heart disease [28-31], blood pressure [32], stroke [33-35], and cancer [36,37]. However, contrary to these previous studies, the clustering approach used in this study allowed to account for well-known collinearities between biomarkers and thus promotes a more holistic perspective. Specifically, findings build on previous studies suggesting a link between inflammation and diseases [25] by demonstrating that it might not be one specific biomarker but a specific biochemical pattern (i.e., high CRP, IL-6, fibrinogen coupled with low cortisol and creatinine) that is associated with diseases. This idea is supported by the observation that individuals in cluster 2, descriptively, indicate a higher disease burden than individuals above the clinically well-established CRP cutoff.

Interestingly, we found no differences in the ORs for PUD between clusters despite the role of inflammation in its pathology [38]. Future research may aim to further examine the role of inflammatory signaling in the pathology of PUD.

While the cluster with high levels of CRP, IL-6, and fibrinogen can be considered a high-risk cluster, cluster 3 with high levels of cortisol and creatinine but low inflammation may be considered a protective cluster in MIDUS. We found that ORs for most diseases were lower in cluster 3 compared to the high-risk cluster but also as compared to cluster 1 with average levels of all biomarkers. Concerning cancer, this difference became significant, potentially suggesting a protective character of this cluster. This would be in contrast to studies suggesting a link between hypercortisolism and disease outcomes $[39,40]$. However, the combination of low inflammation and high cortisol and creatinine as in cluster 3 might indicate the integrity of the glucocorticoid negative feedback system, protecting from negative health outcomes [41]. Longitudinal studies may examine the consequences of this specific biochemical pattern. Towards this aim, we will examine MIDUS follow-up data (10 years after biomarker assessments) with respect to mortality outcomes.

In MIDJA, cluster 2 only seems to be a high-risk cluster for stroke and cancer while for other considered diseases, cluster 1 or cluster 3 indicate the highest burden. One aspect to consider here is that the MIDJA sample ( $N=378)$ and especially cluster 2 were very small in size $(N=30)$. It is, therefore, possible that the present findings lack reliability. However, different biochemical patterns may be associated with different outcomes in the Japanese compared to the U.S. American population because moderating mechanisms such as BMI, nutrition, and medication differ between populations [41]. This idea is supported by the finding that although in both MIDUS and MIDJA, approximately $8 \%$ of participants were assigned to cluster 2, the disease burden in MIDJA was much lower compared to MIDUS. This highlights the importance of individual aspects in disease susceptibility mentioned above and the role of interactions among different cultural, lifestyle and biochemical factors; while an assignment of a U.S. American individual to cluster 2 might be associated with a high disease burden, this might not be the case for a Japanese individual with the similar biochemical profile. Future studies should aim to examine the found biochemical clusters in other cultural contexts promoting a better understanding of their associative and 
predictive character in multiple populations. From a preventive perspective, this may also help to further precise targeted prevention, that is, to better understand which biochemical profile is associated with what disease susceptibility under what conditions.

Limitations. Our work has several strengths such as the validation of the clusters in an independent, Japanese sample and the representative character of cohorts. Yet, the findings face limitations. First, the present study is cross-sectional not allowing causal inferences. Second, the MIDJA sample size was relatively small. It is, therefore, possible that ORs lack reliability. Third, methodological inconsistencies (urine cortisol and creatinine levels in MIDUS, average saliva levels of cortisol and blood levels of creatinine in MIDJA) between the cohorts may have impacted the clustering process. Forth, diseases were assessed via self-report, which bears the risk of a report bias.

Conclusion. While the interactions among biomarkers make the distinction of their outcomes challenging, the design of the current study helps to gain a better understanding regarding the biochemical patterns are present in the general population and how these patterns contribute to different physiological states on a systemic scale. We identified and replicated three distinct biochemical signatures in two mid-life populations including one cluster with collinearly occurring elevated levels of CRP, fibrinogen, and IL- 6 as well as low levels of cortisol and creatinine that indicated the highest prevalence of stroke and cancer.

Future longitudinal studies should aim to test the predictive character of the clusters found in this study, because, if clusters are indeed predictive in terms of risk evaluation, then they would represent a valuable clinical tool for both diagnostics and prevention of diseases. Specifically, if high-risk individuals can be identified by the clustering approach presented here, then these individuals could be provided with personalized treatment options including psychotherapy, e.g., in cases where CM is prevalent, antiinflammatory drugs, or treatment supplements, e.g., nutrition and exercise plans.

\section{Study 2}

\section{Introduction}

Childhood maltreatment (CM) is an umbrella term that includes any act of emotional, physical, and sexual abuse as well as emotional and physical neglect experienced until the age of 18 [42]. CM can have a myriad of negative effects on survivors' mental and somatic health. The association between $\mathrm{CM}$ and inflammation is well established and may underlie the increased prevalence of somatic and mental disorders in CM-exposed individuals [16,43-45]. Thus, $\mathrm{CM}$, which is still an underestimated phenomenon in somatic/clinical settings, might be a disruptive factor in the context of both personalized medicine and targeted prevention, as it may amplify and interact with other disease susceptibility factors, resulting in a massive increase and expansion of an individual's disease risk and development. Therefore, in study 2, the association of $\mathrm{CM}$ with disease prevalence as well as with the assignment to the biochemical clusters was investigated. 
We used the MIDUS sample for these analyses, as CM was not assessed in MIDJA. Based on previous literature, we expected to find higher exposure of CM in clusters with high inflammation as compared to clusters with low inflammation [16,43-45].

\section{Methods}

\section{Assessment of Childhood Maltreatment}

CM was assessed using the Childhood Trauma Questionnaire (CTQ; Bernstein \& Fink, 1998). As a retrospective self-report measure with 28 items, the CTQ assesses five types of CM: Emotional, physical, and sexual abuse, emotional, and physical neglect as well as the tendency to minimize CM [46].

\section{Statistical Analyses}

Cut-off values for moderate CM exposure were used to create dichotomous variables for each CTQ subscale (emotional abuse $\geq 13$; physical abuse $\geq 10$; sexual abuse $\geq 8$; emotional neglect $\geq 15$; and physical neglect $\geq 10$ ) [46]. A composite variable was then computed indicating exposure to at least one category of moderate to severe abuse or neglect $(\mathrm{CM}+)$ vs. no or low exposure $(\mathrm{CM}-)$ [46]. Using the moderate cutoff variable, prevalences of CM were calculated for the whole sample. Next, we compared general disease burden as well as the prevalence of specific diseases in individuals without and with $\mathrm{CM}$ experiences using $c^{2}$ tests and t-tests. Then, a continuous total score of the CTQ was calculated by summing up the scores across all items. This continuous score was used to create a General Linear Model (GLM) with pairwise comparisons correcting for sex, age, BMI, physical activity, alcohol and smoking habits as well as for multiple testing (Bonferroni) comparing CM among clusters. To avoid issues resulting from heteroscedastic residual variances, we performed a bootstrapping (10,000 samples). Bootstrapping, which allows finding robust parameter estimates (i.e., independently from the homoscedasticity assumption of residual variances), is considered the Gold standard approach since our clusters are stable and since none of the covariates included in the GLM is involved in the clustering process [47].

\section{Results}

One-third (36.1\%) reported at least moderate CM on at least one CTQ subscale. Individuals exposed to $\mathrm{CM}$ had a higher overall disease burden with $1.12(S D=1.03)$ diagnoses on average compared to .85 $(S D=.93)$ diagnoses in individuals without CM history $(t(1192)=-4.549, p<.001)$. This difference was mainly driven by the higher prevalence of depression in CM-exposed individuals (36.2\%) compared to individuals without $\mathrm{CM}\left(16.9 \%, c^{2}(1)=61.72, p<.001\right)$.

CM exposure differed between biochemical clusters, with $45.1 \%$ of individuals in cluster 2 reporting at least moderate CM on at least one of the CTQ subscales (28.4\% without CM), compared to $35.9 \%$ in cluster 1 (37.1\% without CM) and $30.8 \%$ in cluster 3 (43.6\% individuals without CM). GLMs using the 
continuous CM score indicated (SI Table 13) the highest CM exposure in cluster 2, followed by clusters 1 and 3 (all $p s<.001)$.

\section{Discussion}

The CM prevalences found here are in line with meta-analytic findings [48] as well as the result that CMexposed individuals have a higher disease burden compared to non-exposed individuals is supported by previous evidence [49-51]. Given the association of CM to inflammatory processes [16,43-45], one mechanism possibly linking $\mathrm{CM}$ to diseases might be the biochemical clusters from study 1 . As we found that cluster 2 had the highest CM exposure and also the highest disease prevalences, specific biochemical profiles may underlie the association between $\mathrm{CM}$ and disease burden. If that is the case, clusters may represent a future leverage point for targeted prevention, enabling CM-exposed individuals to overcome the abusive experience and their stress burden-related health consequences through e.g. psychotherapy and support groups before it comes to the onset and manifestation in the form of severe disease. However, this idea faces the limitation that we could not statistically test this mediation of the biochemical clusters in the link between $\mathrm{CM}$ and disease prevalences as both the possible mediator (clusters) and the dependent variables (disease yes/no) were categorial. To get a deeper insight into this issue, our aim with the MIDUS follow-up data (10 years after biomarker assessments) is to examine whether CM-exposed individuals in cluster 2 indeed show more detrimental outcomes than CM-exposed individuals in the other two clusters.

Limitations. The present findings should be considered in light of the limitation that we used retrospective self-reported measures of $\mathrm{CM}$. Therefore, report and memory biases are possible. Although the value of self-reported measures of CM when investigating its correlates and outcomes has been emphasized [52], future studies should also aim to relate $\mathrm{CM}$ assessed via official reports and compare the relations to biochemical clusters and diseases. CM was not available in the Japanese cohort, therefore, the associative nature of $\mathrm{CM}$ with the identified clusters in the U.S. sample needs future replication in independent cohorts. As this study was cross-sectional, causal inferences cannot be drawn without subsequent research.

Conclusion. Findings complement existing literature indicating detrimental longer-term implications of $\mathrm{CM}$ on survivors' health. Results highlight the importance of identifying $\mathrm{CM}$ as early as possible before it manifests itself biologically and possibly increases disease vulnerability. We thus encourage professionals in preventive and medical care contexts to be attentive to reports of $\mathrm{CM}$ and to consider these in individual treatments; validated screening instruments are available in multiple languages (e.g., CTQ) [46].

\section{Summary And Concluding Discussion}

Our findings suggest three distinct biochemical signatures that are replicable and interculturally stable. One of them is a high-risk cluster indicated by its high disease burden. Due to the cross-sectional character of this study, it might also be that the biochemical clusters are consequences of diseases, 
however, study 2 demonstrating a strong link between the high-risk cluster and CM provides first hints that the clusters could be indeed pre-disease markers affecting the vulnerability to diseases. Future studies should aim to test the predictive character of clusters to evaluate their applicability as pre-disease markers. Further, integrating CM screenings in standard medical practice may be a promising way for identifying individuals at risk and for developing tailored prevention and intervention.

\section{Expert Recommendations}

The assessment of CRP, IL-6, fibrinogen, cortisol, and creatinine should be mandatory in all 3PM (i.e., personalized medicine, targeted prevention, and predictive diagnostics) disciplines to get a global insight into an individual's current health condition. High inflammatory signaling coupled with low compensation, that is, with low cortisol and creatinine, is a detrimental biochemical profile associated with a high disease burden and should be taken as a reason for further examination (especially with respect to artery condition/stroke and cancer) and personalized treatments involving anti-inflammatory drugs, nutrient substitutions, and treatment supplements, e.g., nutrition and exercise plans. Furthermore, individuals with this biochemical profile should be examined with a special focus on early life stress and especially CM. In cases where CM is prevalent, its role in the patient's individual condition pattern should be examined thoroughly and psychotherapy or other stress reducing interventions should be offered/employed.

Future research should examine the predictive character of the found biochemical clusters with respect to long-term well-being, mental and physical health, as well as mortality. Ideally, these studies should examine different cultures promoting a better understanding of the generalizability and limitedness of the predictive power of the found biochemical clusters. Furthermore, this future research may suggest additional factors to be taken into account together with the biochemical clusters, helping to advance and precise disease prediction and, hence, to improve both targeted prevention and personalized interventions.

\section{Declarations}

Funding: None.

Disclosure: The authors report no financial relationships with commercial interests and nothing to disclose.

Availability of data and material: All analyzed data is available publicly: http://www.midus.wisc.edu/data/index.php

Code availability: Not applicable

Ethics approval: The Midlife in the United States Study data collection was reviewed and approved by the Education and Social/Behavioral Sciences and the Health Sciences IRBs at the University of Wisconsin- 
Madison. The Midlife in Japan Study was approved by the IRB of the University of Tokyo.

Consent to participate: Informed consent was obtained by all participants in both the Midlife in the United States Study and in the Midlife in Japan Study.

Consent to publish: After reviewing the manuscript, all authors agreed with its publication in the current form.

\section{References}

1. Buttorff C, Ruder T, Bauman M. Multiple chronic conditions in the United States [Internet]. RAND Corporation; 2017 [cited 2021 May 4]. Available from: http://www.rand.org/pubs/tools/TL221.html.

2. Martin AB, Hartman M, Lassman D, Catlin A, The National Health Expenditure Accounts Team. National Health Care spending in 2019: Steady growth for the fourth consecutive year: Study examines national health care spending for 2019. Health Aff. 2021;40:14-24.

3. Ryff CD, Seeman T, Weinstein M. Midlife in the United States (MIDUS 2): Biomarker Project, 20042009: Version 9 [Internet]. Inter-University Consortium for Political and Social Research; 2010 [cited 2021 Mar 27]. Available from:

https://www.icpsr.umich.edu/icpsrweb/NACDA/studies/29282/versions/V9.

4. Global Burden of Disease Collaborative Network. Global Burden of Disease Study 2017 (GBD 2017) Results. Seattle: Institute for Health Metrics and Evaluation (IHME); 2018.

5. Benjamin EJ, Virani SS, Callaway CW, Chamberlain AM, Chang AR, Cheng S, et al. Heart disease and stroke statistics-2018 Update: A report from the American Heart Association. Circulation [Internet]. 2018 [cited 2021 May 4];137. Available from: https://www.ahajournals.org/doi/10.1161/CIR.0000000000000558.

6. Golubnitschaja O, Liskova A, Koklesova L, Samec M, Biringer K, Büsselberg D, et al Caution, "normal" BMI: Health risks associated with potentially masked individual underweight-EPMA Position Paper 2021. EPMA Journal [Internet]. 2021 [cited 2021 Aug 18]; Available from: https://link.springer.com/10.1007/s13167-021-00251-4.

7. Lopizzo N, Bocchio Chiavetto L, Cattane N, Plazzotta G, Tarazi Fl, Pariante CM, et al. Genenvironment interaction in Major Depression: Focus on experience-dependent biological systems. Front Psychiatry [Internet]. 2015 [cited 2021 Aug 18];6. Available from: http://www.frontiersin.org/Schizophrenia/10.3389/fpsyt.2015.00068/abstract.

8. Huang T, Hu FB. Gene-environment interactions and obesity: Recent developments and future directions. BMC Med Genomics. 2015;8:2.

9. McEwen BS. Stress, adaptation, and disease: Allostasis and allostatic load. Ann N Y Acad Sci. 1998;840:33-44.

10. Chen E, Miller GE, Lachman ME, Gruenewald TL, Seeman TE. Protective factors for adults from lowchildhood socioeconomic circumstances: The benefits of shift-and-persist for allostatic load. 
Psychosom Med. 2012;74:178-86.

11. Gallo LC, Fortmann AL, Mattei J. Allostatic load and the assessment of cumulative biological risk in biobehavioral medicine: Challenges and opportunities. Psychosom Med. 2014;76:478-80.

12. Juster R-P, McEwen BS, Lupien SJ. Allostatic load biomarkers of chronic stress and impact on health and cognition. Neuroscience Biobehavioral Reviews. 2010;35:2-16.

13. Tanaka M. Improving obesity and blood pressure. Hypertens Res. 2020;43:79-89.

14. McEwen BS. Stress and the individual: Mechanisms leading to disease. Arch Intern Med. 1993;153:2093.

15. Thompson AMS, Zanobetti A, Silverman F, Schwartz J, Coull B, Urch B, et al. Baseline repeated measures from controlled human exposure studies: Associations between ambient air pollution exposure and the systemic inflammatory biomarkers IL-6 and fibrinogen. Environ Health Perspect. 2010;118:120-4.

16. Baumeister D, Akhtar R, Ciufolini S, Pariante CM, Mondelli V. Childhood trauma and adulthood inflammation: a meta-analysis of peripheral C-reactive protein, interleukin-6 and tumour necrosis factor-a. Mol Psychiatry. 2016;21:642-9.

17. Rückerl R, Greven S, Ljungman P, Aalto P, Antoniades C, Bellander T, et al. Air pollution and inflammation (interleukin-6, C-reactive protein, fibrinogen) in myocardial infarction survivors. Environ Health Perspect. 2007;115:1072-80.

18. Kashani K, Rosner MH, Ostermann M. Creatinine. From physiology to clinical application. Eur J Intern Med. 2020;72:9-14.

19. Zorn JV, Schür RR, Boks MP, Kahn RS, Joëls M, Vinkers CH. Cortisol stress reactivity across psychiatric disorders: A systematic review and meta-analysis. Psychoneuroendocrinology. 2017;77:25-36.

20. Crimmins EM, Vasunilashorn S, Kim JK, Hagedorn A, Saito Y. A comparison of biological risk factors in two populations: The United States and Japan. Popul Dev Rev. 2008;34:457-82.

21. Gruenewald TL, Karlamangla AS, Hu P, Stein-Merkin S, Crandall C, Koretz B, et al. History of socioeconomic disadvantage and allostatic load in later life. Soc Sci Med. 2012;74:75-83.

22. Kobayashi H, Miyazaki Y. Distribution characteristics of salivary cortisol measurements in a healthy young male population. J Physiol Anthropol. 2015;34:30.

23. Franklin J. The elements of statistical learning: data mining, inference and prediction. The Mathematical Intelligencer. 2005;27:83-5.

24. Pearson TA, Mensah GA, Alexander RW, Anderson JL, Cannon RO, Criqui M, et al. Markers of inflammation and cardiovascular disease: Application to clinical and public health practice: $A$ statement for healthcare professionals from the centers for disease control and prevention and the American Heart Association. Circulation. 2003;107:499-511.

25. Chung HY, Cesari M, Anton S, Marzetti E, Giovannini S, Seo AY, et al. Molecular inflammation: Underpinnings of aging and age-related diseases. Ageing Research Reviews. 2009;8:18-30. 
26. Gimeno D, Kivimäki M, Brunner EJ, Elovainio M, De Vogli R, Steptoe A, et al. Associations of Creactive protein and interleukin-6 with cognitive symptoms of depression: 12-year follow-up of the Whitehall II study. Psychol Med. 2009;39:413-23.

27. Toker S, Shirom A, Shapira I, Berliner S, Melamed S. The association between burnout, depression, anxiety, and inflammation biomarkers: C-reactive protein and fibrinogen in men and women. $\mathrm{J}$ Occup Health Psychol. 2005;10:344-62.

28. Danesh J. Low grade inflammation and coronary heart disease: prospective study and updated meta-analyses. BMJ. 2000;321:199-204.

29. Danesh J, Wheeler JG, Hirschfield GM, Eda S, Eiriksdottir G, Rumley A, et al. C-reactive protein and other circulating markers of inflammation in the prediction of coronary heart disease. $\mathrm{N}$ Engl $\mathrm{J}$ Med. 2004;350:1387-97.

30. Danesh J, Collins R, Appleby P, Peto R. Association of fibrinogen, C-reactive protein, albumin, or leukocyte sount with coronary heart disease: Meta-analyses of prospective studies. JAMA. 1998;279:1477.

31. Luc G, Bard J-M, Juhan-Vague I, Ferrieres J, Evans A, Amouyel P, et al. C-reactive protein, interleukin-6, and fibrinogen as predictors of coronary heart disease: The PRIME study. ATVB. 2003;23:1255-61.

32. Piché M-È, Lemieux S, Weisnagel SJ, Corneau L, Nadeau A, Bergeron J. Relation of high-sensitivity Creactive protein, interleukin-6, tumor necrosis factor-alpha, and fibrinogen to abdominal adipose tissue, blood pressure, and cholesterol and triglyceride levels in healthy postmenopausal women. The American Journal of Cardiology. 2005;96:92-7.

33. Zhou Y, Han W, Gong D, Man C, Fan Y. Hs-CRP in stroke: A meta-analysis. Clin Chim Acta. 2016;453:21-7.

34. Welsh P, Lowe GDO, Chalmers J, Campbell DJ, Rumley A, Neal BC, et al. Associations of proinflammatory cytokines with the risk of recurrent stroke. Stroke. 2008;39:2226-30.

35. Di Napoli M, Papa F, Bocola V. Prognostic influence of increased C-reactive protein and fibrinogen levels in ischemic stroke. Stroke. 2001;32:133-8.

36. Allin $\mathrm{KH}$, Nordestgaard BG. Elevated C-reactive protein in the diagnosis, prognosis, and cause of cancer. Crit Rev Clin Lab Sci. 2011;48:155-70.

37. Qian S, Golubnitschaja O, Zhan X. Chronic inflammation: key player and biomarker-set to predict and prevent cancer development and progression based on individualized patient profiles. EPMA Journal. 2019;10:365-81.

38. Lanas A, Chan FKL. Peptic ulcer disease. The Lancet. 2017;390:613-24.

39. Min Le. Functional hypercortisolism, visceral obesity, and metabolic syndrome. Endocr Pract. 2016;22:506-8.

40. Steffensen C, Pereira AM, Dekkers OM, Jørgensen JOL. Diagnosis of endocrine disease: Prevalence of hypercortisolism in type 2 diabetes patients: a systematic review and meta-analysis. Eur $\mathrm{J}$ Endocrinol. 2016;175:R247-53. 
41. Kalat JW. Biological psychology. 10th ed. Belmont: Wadsworth, Cengage Learning; 2009.

42. Gilbert R, Widom CS, Browne K, Fergusson D, Webb E, Janson S. Burden and consequences of child maltreatment in high-income countries. The Lancet. 2009;373:68-81.

43. Coelho R, Viola TW, Walss-Bass C, Brietzke E, Grassi-Oliveira R. Childhood maltreatment and inflammatory markers: a systematic review. Acta Psychiatr Scand. 2014;129:180-92.

44. Danese A, Moffitt TE, Pariante CM, Ambler A, Poulton R, Caspi A. Elevated inflammation levels in depressed adults with a history of childhood maltreatment. Arch Gen Psychiatry. 2008;65:409.

45. Osborn M, Widom CS. Do documented records and retrospective reports of childhood maltreatment similarly predict chronic inflammation? Psychol Med. 2020;50:2406-15.

46. Bernstein D, Fink L. Manual for the childhood trauma questionnaire. The Psychological Corporation; 1998.

47. Efron B, Tibshirani R. An introduction to the bootstrap. New York: Chapman \& Hall; 1993.

48. Stoltenborgh M, Bakermans-Kranenburg MJ, Alink LRA, van IJzendoorn MH. The prevalence of child maltreatment across the globe: Review of a series of meta-analyses: Prevalence of child Maltreatment across the globe. Child Abuse Rev. 2015;24:37-50.

49. Basu A, McLaughlin KA, Misra S, Koenen KC. Childhood maltreatment and health impact: The examples of cardiovascular disease and type 2 diabetes mellitus in adults. Clin Psychol Sci Pract. 2017;24:125-39.

50. Batten SV, Aslan M, Maciejewski PK, Mazure CM. Childhood maltreatment as a risk factor for adult cardiovascular disease and depression. J Clin Psychiatry. 2004;65:249-54.

51. Hovdestad WE, Shields M, Shaw A, Tonmyr L. Childhood maltreatment as a risk factor for cancer: findings from a population-based survey of Canadian adults. BMC Cancer. 2020;20:70.

52. Danese A, Widom CS. Objective and subjective experiences of child maltreatment and their relationships with psychopathology. Nat Hum Behav. 2020;4:811-8.

\section{Figures}




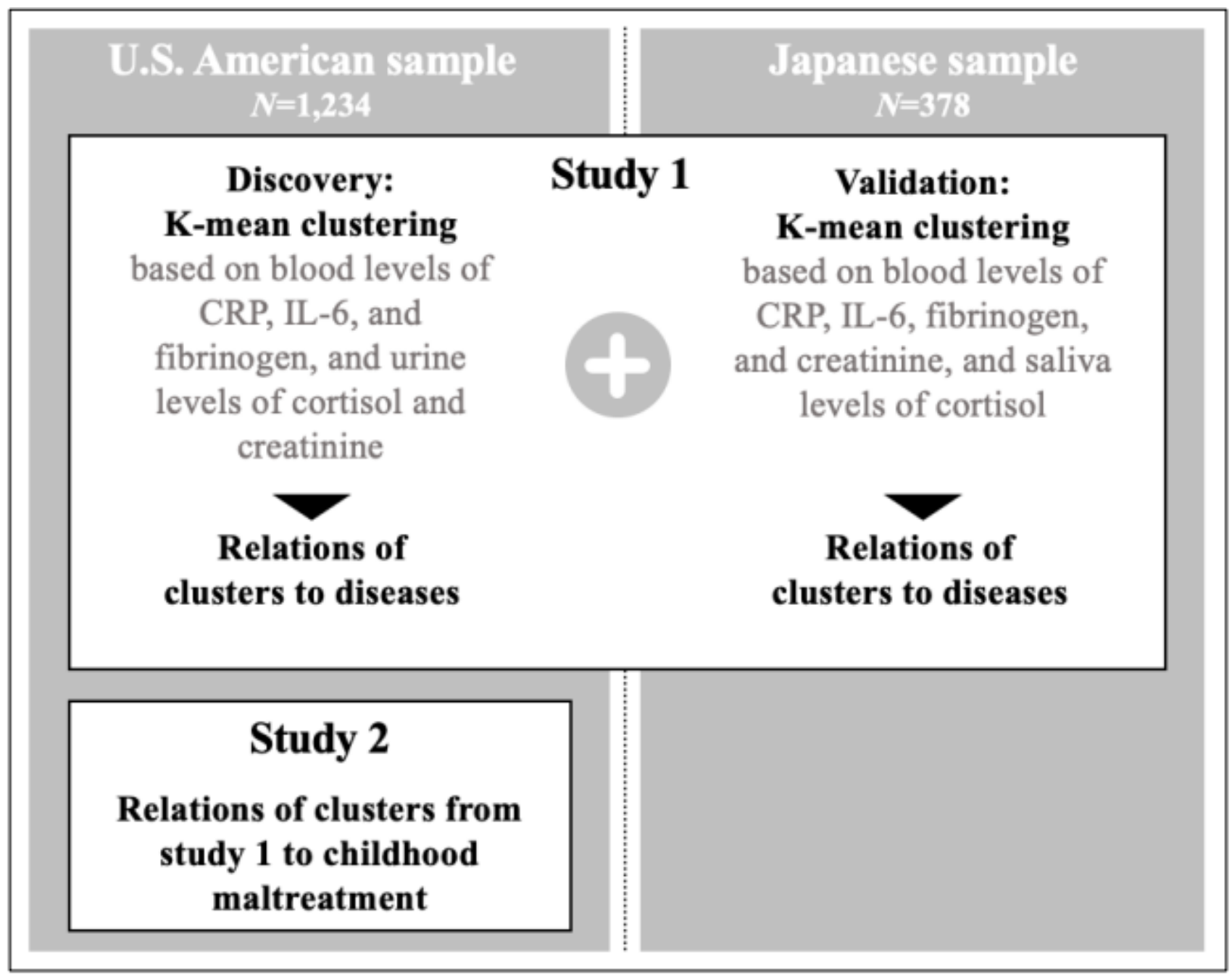

Figure 1

Study Workflow Chart. Note: CRP=C-reactive protein, IL-6=Interleukin-6. All one time measures except saliva cortisol in the Japanese sample which was averaged across three time-points (morning, noon, evening) for a total of three days. 


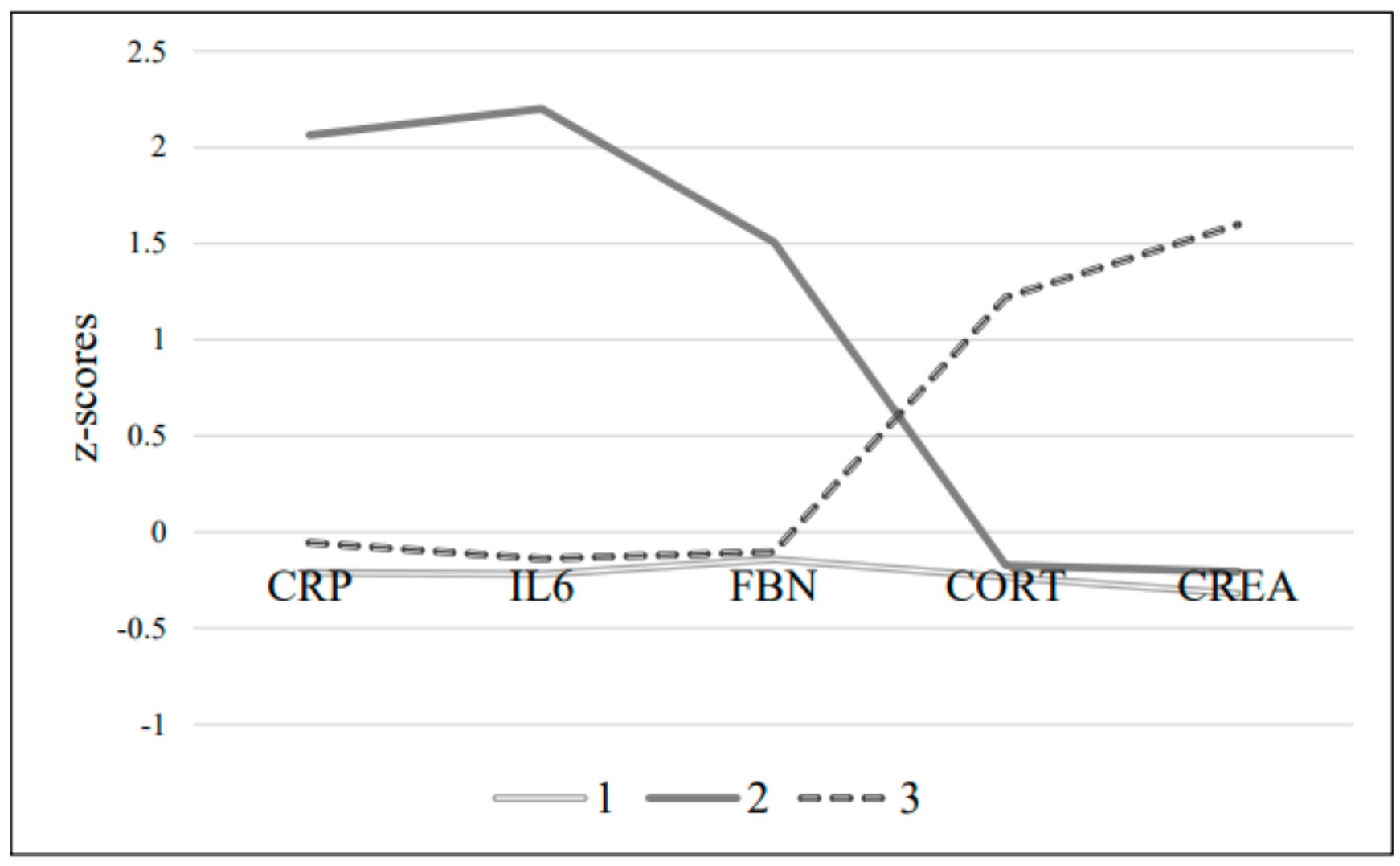

Figure 2

MIDUS: Biochemical Markers (Z-Scores) and Resulting Clusters 1-3. Note: CRP=C-reactive protein ( $\mathrm{ug} / \mathrm{mL}), \mathrm{IL}-6=$ Interleukin-6 $(\mathrm{pg} / \mathrm{mL})$ and FBN=Fibrinogen $(\mathrm{mg} / \mathrm{dL})$ were measured in blood, cortisol $(\mathrm{ug} / \mathrm{dL})$ and creatinine $(\mathrm{mg} / \mathrm{dL})$ were measured in urine. Ncluster1 $=937$, Ncluster2 $=102$, Ncluster3 $=$ 195. 


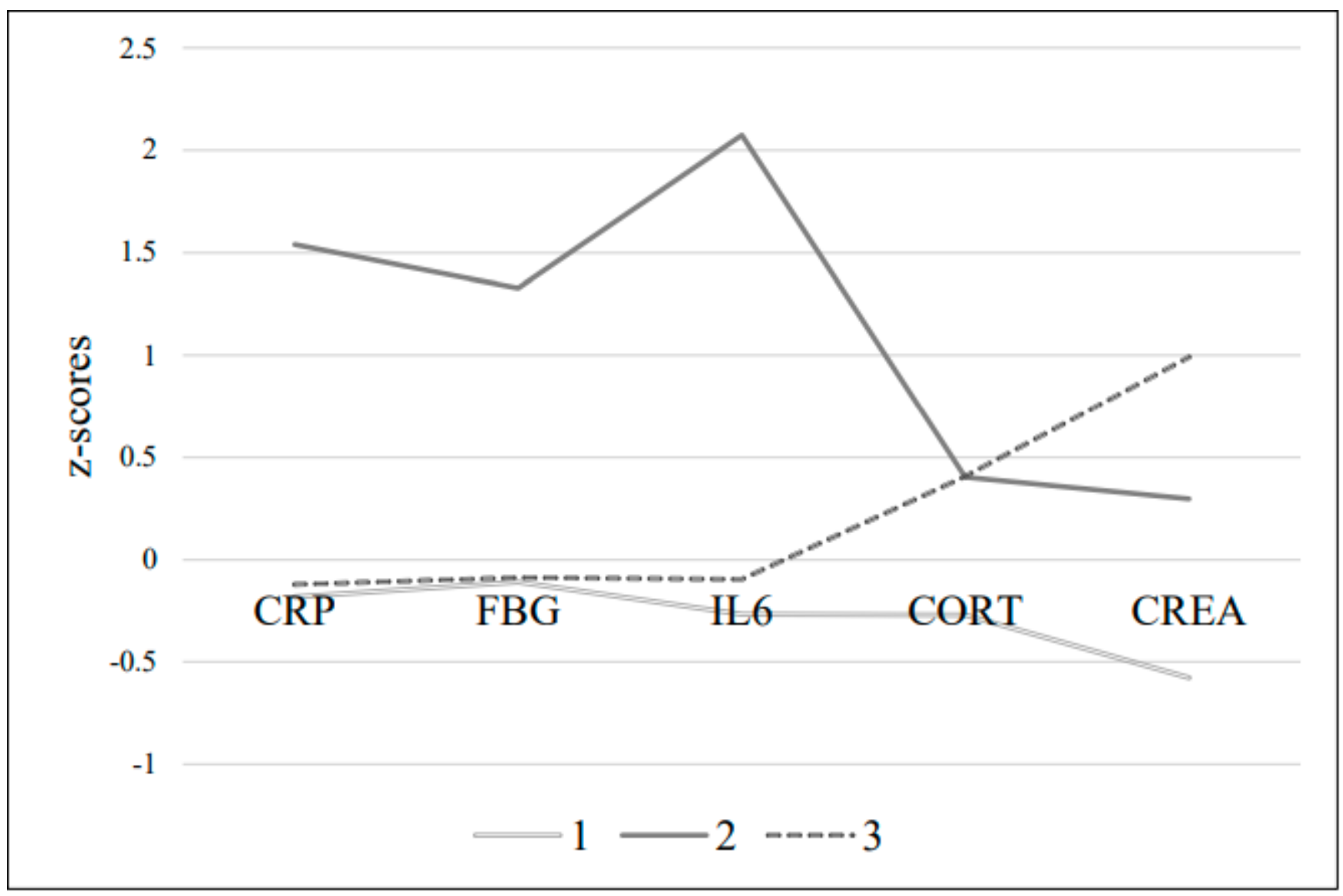

\section{Figure 3}

MIDJA: Biochemical Markers (Z-Scores) and Resulting Clusters 1-3. Note: $\mathrm{CRP}=\mathrm{C}$-reactive protein (ug/mL), IL-6=Interleukin- $6(\mathrm{pg} / \mathrm{mL})$ and FBN=Fibrinogen $(\mathrm{mg} / \mathrm{dL})$, and creatinine $(\mathrm{mg} / \mathrm{dL})$ were measured in blood, cortisol (ug/dL) was measured in saliva. Ncluster1 $=233$, Ncluster2 $=30$, Ncluster3=115. 


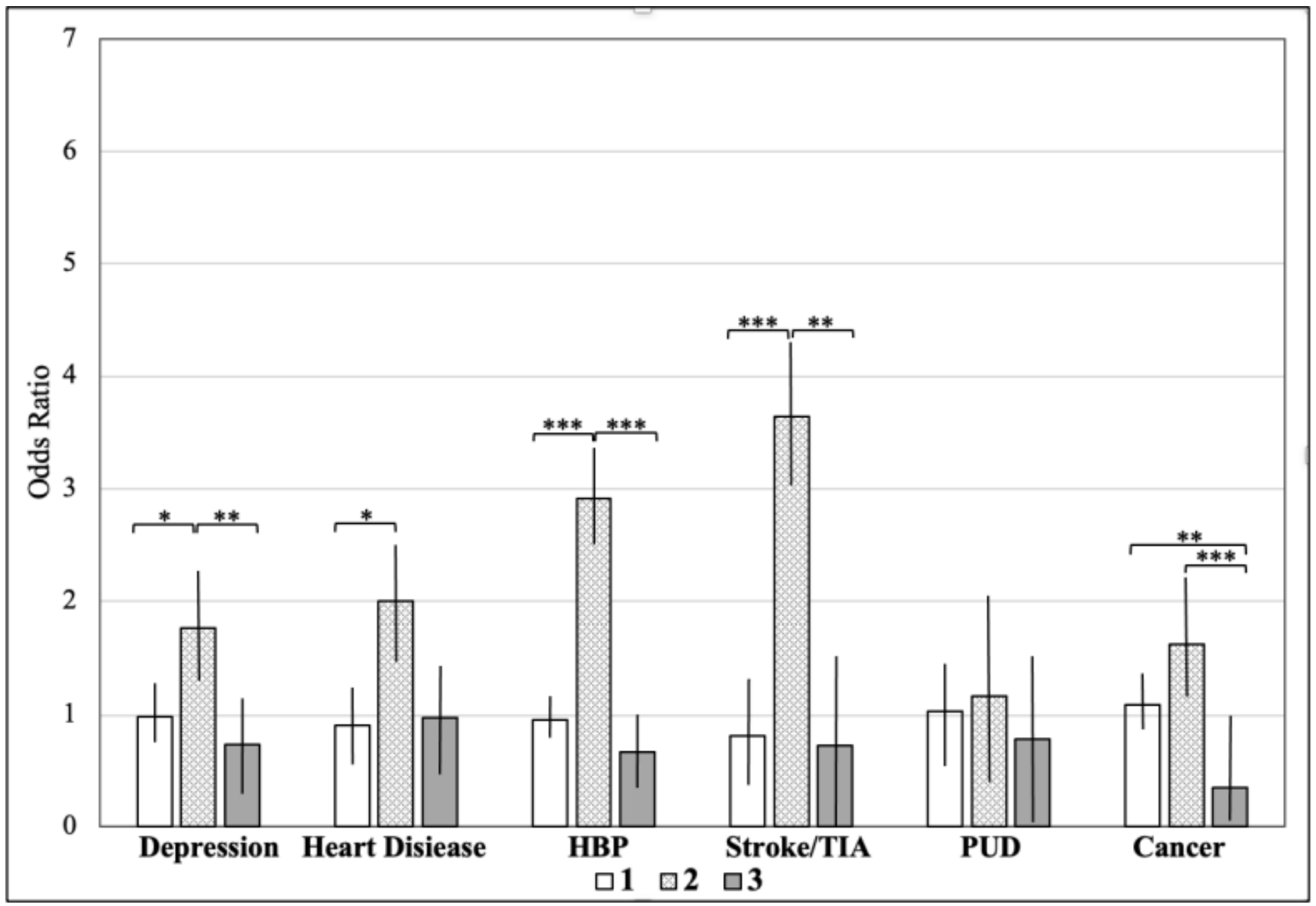

Figure 4

MIDUS: Odds Ratios for Diseases by Cluster. Note: MIDUS=Midlife in the United States sample, HPB=High Blood Pressure, TIA=Transient Ischemic Attack, PUD=Peptic Ulcer Disease. Error bars display 95\% confidence intervals. Comparisons of odds ratios were conducted with log odds ratios using z-tests. * $\mathrm{p}<.05, * \star \mathrm{p}<.01, * \star \star \mathrm{p}<.001, \mathrm{p}$-values are controlled for multiple testing according to Bonferroni. All twotailed. 


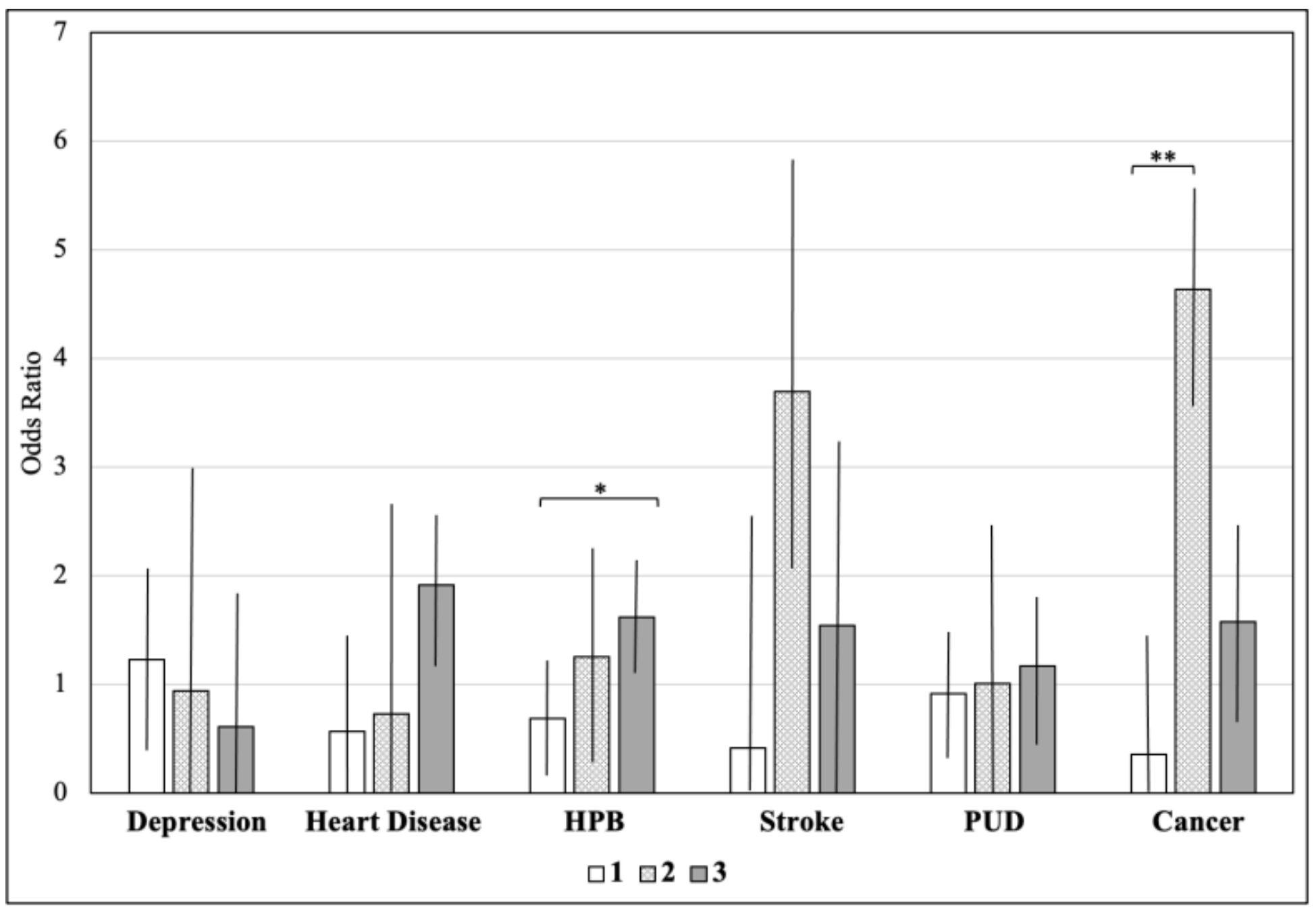

Figure 5

MIDJA: Odds Ratios for Diseases by Cluster. Note: MIDJA=Midlife in Japan sample, HPB=High Blood Pressure, TIA=Transient Ischemic Attack, PUD=Peptic Ulcer Disease. Error bars display 95\% confidence intervals. Comparisons of odds ratios were conducted with log odds ratios using z-tests. ${ }^{\star} p<.05,{ }^{\star *} p<.01$, $\star \star \star p<.001, p$-values are controlled for multiple testing according to Bonferroni. All two-tailed.

\section{Supplementary Files}

This is a list of supplementary files associated with this preprint. Click to download.

- Supplementalmaterial.docx 